\title{
An Optimal Estimate for the Time Singular Limit of an Abstract Wave Equation
}

\author{
By \\ Ralph Chill and Alain Haraux \\ (Universität Ulm, Germany and Université Paris VI, France)
}

\begin{abstract}
R. Ikehata recently proved some integral estimate for the difference between the solution of an abstract heat equation and the solution of an abstract wave equation which results from the heat equation by a time singular perturbation. The estimate is obtained if the initial values are chosen appropriately. We prove a pointwise estimate which improves the above result for large times into several directions, and we also establish the optimality of this estimate for the wave equation in an exterior domain. Our proofs rely on the spectral theorem for unbounded self-adjoint operators.

Key Words and Phrases. Heat equation, Wave equation, Time singular perturbation.

2000 Mathematics Subject Classification Numbers. 34C99.
\end{abstract}

\section{Introduction}

The main objective of this work is to establish a global in time estimate of the difference between a solution $u$ of the abstract dissipative wave equation

$$
\varepsilon u^{\prime \prime}+u^{\prime}+A u=0,
$$

and a related solution $v$ of the abstract heat equation

$$
v^{\prime}+A v=0 .
$$

Here, the operator $(A, D(A))$ is a closed, self-adjoint, positive semidefinite operator on a separable Hilbert space $H$.

Denote by $u_{\varepsilon}$ and $v$, respectively, the mild solutions of the equations (1.1) and (1.2) with initial conditions $u_{\varepsilon}(0)=v(0)=: u^{0} \in V:=D\left(A^{1 / 2}\right)$ and $u_{\varepsilon}^{\prime}(0)=$ : $u^{1} \in H$.

Then one may expect that the solution $u_{\varepsilon}$ of equation (1.1) tends to the solution $v$ of equation (1.2) as $\varepsilon \rightarrow 0+$. J. Kisyński proved that

$$
\forall t \geq 0 \quad\left\|u_{\varepsilon}(t)-v(t)\right\|_{H} \leq \sqrt{\varepsilon}\left\|A^{1 / 2} u^{0}\right\|_{H}+\varepsilon\left\|u^{1}\right\|_{H} ;
$$

see $[9$, Théorème 3.2$]$ or $[6$, Chapter VI]. This estimate implies uniform convergence and gives also the rate of convergence.

Recently, under the additional assumption 


$$
v(0)=u_{\varepsilon}(0)=: u^{0} \in D(A) \quad \text { and } \quad u_{\varepsilon}^{\prime}(0)=: u^{1}=-A u^{0},
$$

$\mathrm{R}$. Ikehata proved in [7] the global integral estimate

$$
\left(\int_{0}^{\infty}\left\|u_{\varepsilon}(t)-v(t)\right\|_{V}^{2} d t\right)^{1 / 2} \leq C \sqrt{\varepsilon}\left\|u^{0}\right\|_{D\left(A^{3 / 2}\right)}
$$

where $C \geq 0$ is a constant independent of $u^{0} \in D\left(A^{3 / 2}\right)$ and $\varepsilon \in(0,1]$.

It is the purpose of this article to prove a pointwise estimate of $\|u(t)-v(t)\|_{V}$ which improves both estimates above for large times $(t \geq 1)$. We obtain in fact a better rate of convergence. For small times, however, the same rates of convergence do not seem to hold.

Throughout this article we will deal with mild solutions of the equations (1.1) and (1.2). Existence of mild solutions for the equations (1.1) and (1.2) can be shown by semigroup methods and is in fact well known.

The main result is the following.

Theorem 1.1 (Global estimate). Let $u_{\varepsilon}$ and $v$, respectively, be the mild solutions of the equations (1.1) and (1.2) with initial conditions as in (1.3). Then there exists a constant $C \geq 0$ independent of $u^{0} \in D(A)$ and $\varepsilon \in(0,1]$ such that

$$
\forall t \geq 1 \quad\left\|u_{\varepsilon}(t)-v(t)\right\|_{V} \leq \frac{C \varepsilon}{t}\left(\left\|u^{0}\right\|_{V}+\sqrt{\varepsilon}\left\|u^{0}\right\|_{D(A)}\right) .
$$

If $A$ is invertible or if 0 is an isolated point in the spectrum $\sigma(A)$, then we have the following, stronger estimate.

Theorem 1.2 (Global exponential estimate). Assume that $A$ is invertible or that $0 \in \sigma(A)$ is an isolated point in $\sigma(A)$. Let $u_{\varepsilon}$ and $v$, respectively, be the mild solutions of the equations (1.1) and (1.2) with initial conditions as in (1.3). Then there exist constants $C \geq 0, \delta>0$ independent of $u^{0} \in D(A)$ and $\varepsilon \in(0,1]$ such that

$$
\forall t \geq 1 \quad\left\|u_{\varepsilon}(t)-v(t)\right\|_{V} \leq \frac{C \varepsilon}{e^{\delta t}}\left(\left\|u^{0}\right\|_{V}+\sqrt{\varepsilon}\left\|u^{0}\right\|_{D(A)}\right) .
$$

Theorem 1.1 is optimal in the following sense.

Theorem 1.3 (Optimality). If 0 belongs to $\sigma(A)$ and if 0 is not isolated in $\sigma(A)$, then

$$
\limsup _{t \rightarrow \infty} \sup _{\varepsilon \in(0,1]} \sup _{\substack{u^{\in} \in D(A) \\\left\|u^{0}\right\|_{D(A)} \leq 1}} \frac{t}{\varepsilon}\left\|u_{\varepsilon}(t)-v(t)\right\|_{H}>0 .
$$

For fixed initial values we have the following individual behaviour. 
Theorem 1.4 (Individual behaviour). Let $u_{\varepsilon}$ and $v$, respectively, be the mild solutions of the equations (1.1) and (1.2) with initial conditions as in (1.3). Then

$$
\lim _{t \rightarrow \infty} \frac{t}{\varepsilon}\left\|u_{\varepsilon}(t)-v(t)\right\|_{V}=0 \quad \text { uniformly in } \varepsilon \in(0,1]
$$

\section{Proof of the main results}

In this and the following section, we denote by $C \geq 0$ a generic constant, the value of which may vary from line to line but does not depend on $u^{0} \in D(A)$ and $\varepsilon \in(0,1]$.

We start by proving the following lemma.

Lemma 2.1. Let $u_{\varepsilon}$ be the mild solution of (1.1) corresponding to the initial values $u(0)=u^{0} \in V$ and $u^{\prime}(0)=u^{1} \in H$. Assume that $A \geq I /(16 \varepsilon)$. Then, for every $t \geq 0$,

$$
\varepsilon\left\|u_{\varepsilon}^{\prime}(t)\right\|_{H}^{2}+\left\|A^{1 / 2} u_{\varepsilon}(t)\right\|_{H}^{2} \leq 4 e^{-t /(36 \varepsilon)}\left(\varepsilon\left\|u^{1}\right\|_{H}^{2}+\left\|A^{1 / 2} u^{0}\right\|_{H}^{2}\right) .
$$

Proof. Assume first that $u_{\varepsilon}$ is in fact a classical solution. Since $\varepsilon \in(0,1]$ is fixed, we may write for simplicity $u:=u_{\varepsilon}$.

Let $\beta>0$ and define for every $t \in \boldsymbol{R}_{+}$

$$
H(t):=\frac{\varepsilon}{2}\left\|u^{\prime}\right\|_{H}^{2}+\frac{1}{2}\left\|A^{1 / 2} u\right\|_{H}^{2}+\beta \varepsilon\left(u, u^{\prime}\right)_{H} .
$$

Then the function $H$ is differentiable, and for every $t \in \boldsymbol{R}_{+}$

$$
\begin{aligned}
\frac{d}{d t} H(t) & =\left(u^{\prime}, \varepsilon u^{\prime \prime}+A u\right)_{H}+\beta \varepsilon\left\|u^{\prime}\right\|_{H}^{2}-\beta\left(u, u^{\prime}+A u\right)_{H} \\
& \leq-(1-\beta \varepsilon)\left\|u^{\prime}\right\|_{H}^{2}-\beta\left(u, u^{\prime}\right)_{H}-\beta\left\|A^{1 / 2} u\right\|_{H}^{2} .
\end{aligned}
$$

From the Cauchy-Schwarz inequality we obtain for every $\lambda>0$

$$
\left|\left(u, u^{\prime}\right)_{H}\right| \leq \lambda\left\|u^{\prime}\right\|_{H}^{2}+\frac{1}{4 \lambda}\|u\|_{H}^{2} \leq \lambda\left\|u^{\prime}\right\|_{H}^{2}+\frac{4 \varepsilon}{\lambda}\left\|A^{1 / 2} u\right\|_{H}^{2}
$$

Hence, if we put

$$
\beta:=\frac{1}{18 \varepsilon} \quad \text { and } \quad \lambda=8 \varepsilon
$$

then we obtain

$$
\frac{d}{d t} H(t) \leq-\frac{1}{18 \varepsilon}\left(\frac{\varepsilon}{2}\left\|u^{\prime}\right\|_{H}^{2}+\frac{1}{2}\left\|A^{1 / 2} u\right\|_{H}^{2}\right)
$$


Moreover,

$$
H(t) \leq \varepsilon\left\|u^{\prime}\right\|_{H}^{2}+\left\|A^{1 / 2} u\right\|_{H}^{2}
$$

and, by using the following variation from (2.1),

$$
\left|\left(u, u^{\prime}\right)_{H}\right| \leq \frac{\lambda}{2}\left\|u^{\prime}\right\|_{H}^{2}+\frac{8 \varepsilon}{\lambda}\left\|A^{1 / 2} u\right\|_{H}^{2},
$$

we find

$$
H(t) \geq \frac{1}{2}\left(\frac{\varepsilon}{2}\left\|u^{\prime}\right\|_{H}^{2}+\frac{1}{2}\left\|A^{1 / 2} u\right\|_{H}^{2}\right)
$$

In particular

$$
\frac{d}{d t} H(t) \leq-\frac{1}{36 \varepsilon} H(t), \quad t \in \boldsymbol{R}_{+} .
$$

Hence,

$$
H(t) \leq e^{-t /(36 \varepsilon)} H(0), \quad t \in \boldsymbol{R}_{+},
$$

so that

$$
\frac{1}{2}\left(\frac{\varepsilon}{2}\left\|u^{\prime}\right\|_{H}^{2}+\frac{1}{2}\left\|A^{1 / 2} u\right\|_{H}^{2}\right) \leq e^{-t /(36 \varepsilon)}\left(\varepsilon\left\|u^{1}\right\|_{H}^{2}+\left\|A^{1 / 2} u^{0}\right\|_{H}^{2}\right), \quad t \in \boldsymbol{R}_{+} .
$$

This implies the claim for classical solutions $u$. The general claim follows from this and by approximation of mild solutions by smooth solutions.

Proof of Theorem 1.1. By the spectral theorem for self-adjoint operators ([13, Theorem VIII.4, p. 260]), the operator $A$ is unitarily equivalent to a multiplication operator on some $L^{2}$ space. Therefore, it is sufficient to consider the case when

$$
\begin{aligned}
H & =L^{2}(E, d \mu), \\
(A u)(x) & =a(x) u(x), \quad x \in E, u \in D(A),
\end{aligned}
$$

where $(E, \mu)$ is a measure space, $a$ is a nonnegative $\mu$-measurable function,

$$
D(A)=\{u \in H: a \cdot u \in H\}=L^{2}\left(E ;\left(1+a^{2}\right) d \mu\right),
$$

and

$$
V=L^{2}(E ;(1+a) d \mu) .
$$

So let $H$ and $A$ be as above, fix $\varepsilon \in(0,1]$, and let $x \in E$.

If $u_{\varepsilon}$ and $v$ are solutions of the equations (1.1) and (1.2), respectively, then 
the functions $u_{\varepsilon}(\cdot, x)$ and $v(\cdot, x)$ (which will in the following for simplicity be denoted by $u$ and $v$ ) satisfy the ordinary differential equations

$$
\varepsilon u^{\prime \prime}+u^{\prime}+a(x) u=0,
$$

and

$$
v^{\prime}+a(x) v=0 .
$$

For the restrictions of the solutions $u_{\varepsilon}$ and $v$ to the region $\{a(x) \geq 1 /(16 \varepsilon)\}$ the claim follows from Lemma 2.1 (see Step 3 below). It is therefore sufficient to consider the case $a(x)=\omega^{2} \leq 1 /(16 \varepsilon)$.

Step 1: Assume that $0 \leq a(x)=\omega^{2} \leq 1 /(16 \varepsilon)$. Then we have the formulas

$$
\begin{aligned}
& v(t)=e^{-\omega^{2} t} u^{0} \quad \text { and } \\
& u(t)=e^{-\omega^{2} t} z(t),
\end{aligned}
$$

with

$$
z(t)=\frac{\sigma_{2} u^{0}-u^{1}}{\sigma_{2}-\sigma_{1}} e^{-\varepsilon \sigma_{1}^{2} t}+\frac{\sigma_{1} u^{0}-u^{1}}{\sigma_{1}-\sigma_{2}} e^{-\varepsilon \sigma_{2}^{2} t}
$$

where

$$
\sigma_{1}=\frac{-1+\sqrt{1-4 \omega^{2} \varepsilon}}{2 \varepsilon} \quad \text { and } \quad \sigma_{2}=\frac{-1-\sqrt{1-4 \omega^{2} \varepsilon}}{2 \varepsilon}
$$

Note that $\sigma_{1}$ and $\sigma_{2}$ are the two solutions of the equation

$$
\varepsilon \sigma^{2}+\sigma+\omega^{2}=0 .
$$

Therefore, using the assumption (1.3),

$$
\begin{aligned}
w(t) & :=u(t)-v(t) \\
& =u^{0} e^{-\omega^{2} t}\left(\frac{\sigma_{2}+\omega^{2}}{\sigma_{2}-\sigma_{1}} e^{-\varepsilon \sigma_{1}^{2} t}-\frac{\sigma_{1}+\omega^{2}}{\sigma_{2}-\sigma_{1}} e^{-\varepsilon \sigma_{2}^{2} t}-1\right) \\
& =u^{0} e^{-\omega^{2} t}\left(\frac{-\varepsilon \sigma_{2}^{2}}{\sigma_{2}-\sigma_{1}}\left(e^{-\varepsilon \sigma_{1}^{2} t}-1\right)-\frac{\sigma_{1}+\omega^{2}}{\sigma_{2}-\sigma_{1}}\left(e^{-\varepsilon \sigma_{2}^{2} t}-1\right)\right) .
\end{aligned}
$$

We have the estimates

$$
\frac{1}{\varepsilon} \geq \sigma_{1}-\sigma_{2}=\frac{\sqrt{1-4 \omega^{2} \varepsilon}}{\varepsilon} \geq \frac{\sqrt{3}}{2 \varepsilon},
$$

and 


$$
\omega^{2} \leq\left|\sigma_{1}\right|=\left|\frac{2 \omega^{2}}{1+\sqrt{1-4 \varepsilon \omega^{2}}}\right| \leq \frac{3}{2} \omega^{2},
$$

and

$$
\frac{1}{2 \varepsilon} \leq\left|\sigma_{2}\right| \leq \frac{1}{\varepsilon}
$$

This implies

$$
\left|\frac{\varepsilon \sigma_{2}^{2}}{\sigma_{2}-\sigma_{1}}\right| \leq \frac{2}{\sqrt{3}} .
$$

Hence, we obtain for every $t \geq 0$

$$
\begin{aligned}
\left|e^{-\omega^{2} t} \frac{\varepsilon \sigma_{2}^{2}}{\sigma_{2}-\sigma_{1}}\left(e^{-\varepsilon \sigma_{1}^{2} t}-1\right)\right| & \leq e^{-\omega^{2} t} \frac{2}{\sqrt{3}} \varepsilon \sigma_{1}^{2} t \\
& \leq \varepsilon \frac{3 \sqrt{3}}{2} \frac{\omega^{4} t^{2} e^{-\omega^{2} t}}{t},
\end{aligned}
$$

and

$$
\begin{aligned}
\left|e^{-\omega^{2} t} \frac{\sigma_{1}+\omega^{2}}{\sigma_{2}-\sigma_{1}}\left(e^{-\varepsilon \sigma_{2}^{2} t}-1\right)\right| & \leq e^{-\omega^{2} t} \frac{5}{2} \omega^{2} \frac{2 \varepsilon}{\sqrt{3}} \\
& \leq \varepsilon \frac{5}{\sqrt{3}} \frac{\omega^{2} t e^{-\omega^{2} t}}{t} .
\end{aligned}
$$

These two estimates are independent of $a(x)=\omega^{2} \leq 1 /(16 \varepsilon)$.

Step 2: In the second step we still assume that $a(x)=\omega^{2} \leq 1 /(16 \varepsilon)$.

Since

$$
\sup _{s \geq 0} s^{2} e^{-s}=\frac{4}{e^{2}} \quad \text { and } \quad \sup _{s \geq 0} s e^{-s}=\frac{1}{e},
$$

the estimates (2.5) and (2.6) from Step 1 imply for every $t>0$

$$
\begin{aligned}
|w(t)| & \leq \frac{\varepsilon}{t}\left|u^{0}\right|\left(\frac{3 \sqrt{3}}{2} \frac{4}{e^{2}}+\frac{5}{\sqrt{3}} \frac{1}{e}\right) \\
& \leq \frac{4 \varepsilon}{t}\left|u^{0}\right| .
\end{aligned}
$$

This estimate is independent of $a(x)=\omega^{2} \leq 1 /(16 \varepsilon)$.

Step 3: Note that for fixed $\varepsilon \in(0,1]$ the space

$$
H_{\varepsilon}:=\left\{f \in H: \operatorname{supp} f \subset\left\{a(x)>\frac{1}{16 \varepsilon}\right\}\right\}
$$


is invariant under the dynamics of equations (1.1) and (1.2). The restriction of the operator $A$ to this space is strictly positive. In fact, $A \geq I /(16 \varepsilon)$.

Lemma 2.1 applied to the orthogonal projection of the solution $u_{\varepsilon}$ to the space $H_{\varepsilon}$ (note that this projection is the multiplication with the characteristic function of the set $\{a(x)>1 /(16 \varepsilon)\})$ implies for every $t \geq 0$

$$
\int_{\{a(x) \geq 1 /(16 \varepsilon)\}}\left|u_{\varepsilon}(t, x)\right|^{2}(1+a(x)) d \mu(x) \leq C e^{-t /(36 \varepsilon)}\left(\left\|A^{1 / 2} u^{0}\right\|_{H}^{2}+\varepsilon\left\|A u^{0}\right\|_{H}^{2}\right) .
$$

Moreover, from standard theory of the linear first order problem follows

$$
\int_{\{a(x) \geq 1 /(16 \varepsilon)\}}|v(t, x)|^{2}(1+a(x)) d \mu(x) \leq e^{-t /(8 \varepsilon)}\left\|u^{0}\right\|_{V}^{2}, \quad t \geq 0 .
$$

Therefore, using the estimate (2.7), we obtain for every $t>0$

$$
\begin{aligned}
\left.\int_{\{a(x)} \geq 1 /(16 \varepsilon)\right\} & \left|u_{\varepsilon}(t, x)-v(t, x)\right|^{2}(1+a(x)) d \mu(x) \\
& \leq C \frac{\varepsilon^{2}}{t^{2}} \frac{t^{2}}{\varepsilon^{2}} e^{-t /(36 \varepsilon)}\left(\left\|u^{0}\right\|_{V}^{2}+\varepsilon\left\|u^{0}\right\|_{D(A)}^{2}\right) \\
& \leq C \frac{\varepsilon^{2}}{t^{2}}\left(\left\|u^{0}\right\|_{V}^{2}+\varepsilon\left\|u^{0}\right\|_{D(A)}^{2}\right) .
\end{aligned}
$$

This estimate and the estimate from Step 2 imply for every $t>0$

$$
\begin{aligned}
\| u_{\varepsilon}(t)- & v(t) \|_{V} \\
= & \left(\int_{E}\left|u_{\varepsilon}(t, x)-v(t, x)\right|^{2}(1+a(x)) d \mu(x)\right)^{1 / 2} \\
\leq & \left(\int_{\{a(x)>1 / 16\}}\left|u_{\varepsilon}(t, x)-v(t, x)\right|^{2}(1+a(x)) d \mu(x)\right)^{1 / 2} \\
& +\left(\int_{\{a(x) \leq 1 / 16\}}\left|u_{\varepsilon}(t, x)-v(t, x)\right|^{2}(1+a(x)) d \mu(x)\right)^{1 / 2} \\
\leq & \frac{C \varepsilon}{t}\left(\left\|u^{0}\right\|_{V}+\sqrt{\varepsilon}\left\|u^{0}\right\|_{D(A)}\right) \\
& +\left(\int_{\{a(x) \leq 1 / 16\}} \frac{C^{2} \varepsilon^{2}}{t^{2}}\left|u^{0}(x)\right|^{2}(1+a(x)) d \mu(x)\right)^{1 / 2} \\
\leq & \frac{C \varepsilon}{t}\left(\left\|u^{0}\right\|_{V}+\sqrt{\varepsilon}\left\|u^{0}\right\|_{D(A)}\right) .
\end{aligned}
$$

This is the claim. 
Proof of Theorem 1.2. In order to prove Theorem 1.2, we can proceed in a way similar to the proof of Theorem 1.1 .

First, by the spectral theorem for self-adjoint operators, we may again assume without loss of generality that $A$ is a multiplication operator, as in the proof of Theorem 1.1.

With this identification, the assumption that $A$ is invertible or that $0 \in \sigma(A)$ is an isolated point in $\sigma(A)$ implies that there exists $\alpha>0$ such that the set

$$
\{0<a(x)<\alpha\}
$$

has $\mu$-measure 0 .

Step 1: Assume that $\alpha \leq a(x)=\omega^{2} \leq 1 /(16 \varepsilon)$, and let $\delta \in(0, \alpha)$.

Then the estimates (2.5) and (2.6) together with (2.7) imply for every $t>0$

$$
\begin{aligned}
|w(t, x)| & =\left|u_{\varepsilon}(t, x)-v(t, x)\right| \\
& \leq \frac{C \varepsilon}{e^{\delta t}}\left|u^{0}\right| \frac{1}{t}\left(\omega^{4} t^{2}+\omega^{2} t\right) e^{-\left(\omega^{2}-\delta\right) t} \\
& \leq \frac{C \varepsilon}{e^{\delta t}}\left|u^{0}(x)\right| \frac{1}{t}
\end{aligned}
$$

In the above estimate we have used that $\omega^{2}-\delta \geq \alpha-\delta>0$. The constant $C \geq 0$ depends on $\alpha$ and $\alpha-\delta$, but it does not depend on $u^{0}$ and $\varepsilon \in(0,1]$.

The above estimate is independent of $\alpha \leq a(x)=\omega^{2} \leq 1 /(16 \varepsilon)$.

Step 2: Assume that $a(x)=\omega^{2}=0$. Then one will easily verify that $u_{\varepsilon}(t, x)=v(t, x)=u^{0}(x)$. Hence,

$$
|w(t, x)|=\left|u_{\varepsilon}(t, x)-v(t, x)\right|=0, \quad t \geq 0 .
$$

Step 3: Proceeding as in the Step 3 of the proof of Theorem 1.1, Lemma 2.1 together with (2.7) implies for every $\delta<1 / 72$ and every $t>0$

$$
\begin{aligned}
\int_{\{a(x) \geq 1 /(16 \varepsilon)\}}\left|u_{\varepsilon}(t, x)-v(t, x)\right|^{2}(1+a(x)) d \mu(x) \\
\leq \frac{C}{e^{2 \delta t}} \frac{\varepsilon^{2}}{t^{2}} \frac{t^{2}}{\varepsilon^{2}} e^{-t(1 /(36 \varepsilon)-2 \delta)}\left(\left\|u^{0}\right\|_{V}^{2}+\varepsilon\left\|u^{0}\right\|_{D(A)}^{2}\right) \\
\leq \frac{C \varepsilon^{2}}{e^{2 \delta t}} \frac{1}{t^{2}}\left(\left\|u^{0}\right\|_{V}^{2}+\varepsilon\left\|u^{0}\right\|_{D(A)}^{2}\right) .
\end{aligned}
$$

This estimate and the estimate from the preceding Steps 1 and 2 imply for every $\delta<\min \{1 / 72, \alpha\}$ and every $t \geq 1$ 


$$
\begin{aligned}
\left\|u_{\varepsilon}(t)-v(t)\right\|_{V}= & \left(\int_{E}\left|u_{\varepsilon}(t, x)-v(t, x)\right|^{2}(1+a(x)) d \mu(x)\right)^{1 / 2} \\
\leq & \left(\int_{\{a(x)>1 / 16\}}\left|u_{\varepsilon}(t, x)-v(t, x)\right|^{2}(1+a(x)) d \mu(x)\right)^{1 / 2} \\
& +\left(\int_{\{a(x) \leq 1 / 16\}}\left|u_{\varepsilon}(t, x)-v(t, x)\right|^{2}(1+a(x)) d \mu(x)\right)^{1 / 2} \\
\leq & \frac{C \varepsilon}{e^{\delta t}}\left(\left\|u^{0}\right\|_{V}+\sqrt{\varepsilon}\left\|u^{0}\right\|_{D(A)}\right) \\
& +\left(\int_{\{\alpha \leq a(x) \leq 1 / 16\}} \frac{C^{2} \varepsilon^{2}}{\left.e^{2 \delta t}\left|u^{0}(x)\right|^{2}(1+a(x)) d \mu(x)\right)^{1 / 2}}\right. \\
\leq & \frac{C \varepsilon}{e^{\delta t}}\left(\left\|u^{0}\right\|_{V}+\sqrt{\varepsilon}\left\|u^{0}\right\|_{D(A)}\right) .
\end{aligned}
$$

This is the claim.

Proof of Theorem 1.3. By the spectral theorem for self-adjoint operators, we may assume that $A$ is a multiplication operator as in the proof of Theorem 1.1 .

With this identification the assumption that $0 \in \sigma(A)$ is not an isolated point in the spectrum $\sigma(A)$ is equivalent to saying that infinitely many of the sets

$$
E_{n}:=\left\{\frac{1}{n+1} \leq a(x)<\frac{1}{n}\right\}
$$

have positive $\mu$-measure.

If $a(x)=\omega^{2} \leq 1 / 16$, then, as we have seen in the proof of Theorem 1.1 ,

$$
\left|u_{\varepsilon}(t)-v(t)\right|=\left|u^{0}\right| e^{-\omega^{2} t}\left|\frac{\varepsilon \sigma_{2}^{2}}{\sigma_{1}-\sigma_{2}}\left(e^{-\varepsilon \sigma_{1}^{2} t}-1\right)-\frac{\varepsilon \sigma_{1}^{2}}{\sigma_{1}-\sigma_{2}}\left(e^{-\varepsilon \sigma_{2}^{2} t}-1\right)\right|
$$

where $u_{\varepsilon}$ and $v$ denote the solutions of the scalar equations of second and first order, respectively.

If $n \geq 16, \omega^{2} \in[1 /(n+1), 1 / n)$ and $t \in[n+1,2 n]$, then $\omega^{2} t \in[1,2]$ and

$$
\frac{t}{\varepsilon}\left|u_{\varepsilon}(t)-v(t)\right| \geq\left|u^{0}\right| \frac{1}{\varepsilon \omega^{2}} e^{-2}\left(\frac{\varepsilon \sigma_{2}^{2}}{\sigma_{1}-\sigma_{2}}\left(1-e^{-\varepsilon \sigma_{1}^{2} t}\right)-\frac{\varepsilon \sigma_{1}^{2}}{\sigma_{1}-\sigma_{2}}\left(1-e^{-\varepsilon \sigma_{2}^{2} t}\right)\right)
$$




$$
\begin{aligned}
& \geq\left|u^{0}\right| \frac{1}{\varepsilon \omega^{2}} e^{-2}\left(\frac{1}{4}\left(1-e^{-\varepsilon \omega^{2}}\right)-\varepsilon \frac{9 \omega^{4}}{4} \frac{2 \varepsilon}{\sqrt{3}}\left(1-e^{-2 /\left(\varepsilon \omega^{2}\right)}\right)\right) \\
& \geq\left|u^{0}\right| e^{-2}\left(\frac{1}{4 \varepsilon \omega^{2}}\left(1-e^{-\varepsilon \omega^{2}}\right)-\varepsilon \omega^{2} \frac{3 \sqrt{3}}{2}\right) .
\end{aligned}
$$

In the above sequence of inequalities we have used the estimates (2.2)-(2.4) from the proof of Theorem 1.1.

Since the function

$$
s \mapsto \frac{1}{4 s}\left(1-e^{-s}\right)-\frac{3 \sqrt{3}}{2} s
$$

is strictly positive on some interval $\left[0,1 / n_{0}\right]\left(n_{0} \geq 16\right)$, we see that there exists a constant $C>0$ such that for every $n \geq n_{0}$ and every $\omega^{2}$ and $t$ as above

$$
\frac{t}{\varepsilon}\left|u_{\varepsilon}(t)-v(t)\right| \geq C\left|u^{0}\right|
$$

Now, let $u_{n}^{0}:=1 / \sqrt{\mu\left(E_{n}\right)} \chi_{E_{n}}$, where $\chi_{E_{n}}$ is the characteristic function of the set $E_{n}$ and $\mu\left(E_{n}\right)>0$. Then $\left\|u_{n}^{0}\right\|_{H}=1$ and $\left\|u_{n}^{0}\right\|_{D(A)} \leq 2$.

Let $u_{\varepsilon}^{n}$ and $v^{n}$ be the solutions of the equations (1.1) and (1.2) corresponding to the initial values $u(0)=v(0)=u_{n}^{0}$ and $u^{\prime}(0)=-A u_{n}^{0}$.

Integrating the above estimate in $x$, we find for every $t \in[n+1,2 n]$

$$
\begin{aligned}
\frac{t}{\varepsilon}\left\|u_{\varepsilon}^{n}(t)-v^{n}(t)\right\|_{H} & =\frac{t}{\varepsilon}\left(\int_{E_{n}}\left|u_{\varepsilon}^{n}(t, x)-v^{n}(t, x)\right|^{2} d \mu(x)\right)^{1 / 2} \\
& \geq C\left(\int_{E_{n}}\left|u_{n}^{0}(x)\right|^{2} d \mu(x)\right)^{1 / 2}=C .
\end{aligned}
$$

The claim follows by letting $n$, and hence $t$, tend to $\infty$.

Proof of Theorem 1.4. By the spectral theorem for self-adjoint operators, we may assume that $A$ is a multiplication operator as in the proof of Theorem 1.1 .

Consider the Banach spaces

$$
X:=\left\{f \in C([1, \infty) \times(0,1] ; V): \sup _{t \geq 1, \varepsilon \in(0,1]} t\|f(t, \varepsilon)\|_{V}<\infty\right\}
$$

and

$$
X_{0}:=\left\{f \in X: \lim _{t \rightarrow \infty} t\|f(t, \varepsilon)\|_{V}=0 \text { uniformly in } \varepsilon \in(0,1]\right\},
$$

both endowed with the natural supremum norm. 
By Theorem 1.1, the linear operator

$$
\begin{aligned}
D(A) & \rightarrow X, \\
u^{0} & \mapsto f_{u^{0}},
\end{aligned}
$$

where $f_{u^{0}}(t, \varepsilon):=\left(u_{\varepsilon}(t)-v(t)\right) / \varepsilon$, and $u_{\varepsilon}$ and $v$ are the two solutions of the equations (1.1) and (1.2), respectively, corresponding to the initial values $u(0)=$ $v(0)=u^{0}$ and $u^{\prime}(0)=-A u^{0}$, is well defined and bounded.

By Theorem 1.2, this operator maps the set

$$
M_{n}:=\left\{u^{0} \in D(A): \operatorname{supp} u^{0} \subset\{a(x) \geq 1 / n\}\right\} \cup(\operatorname{Ker} A)
$$

into $X_{0}$.

Since the union $\bigcup_{n \in N} M_{n}$ is total in $D(A)$, the claim follows.

\section{Applications and Remarks}

3.1. Theorem 1.1 improves the global integral estimate from [7, Theorem 1.1] and the uniform estimates from [9, Théorème 3.2] and [6, Section VI.5, Theorem 5.5] (see also the Introduction) into several directions, at least for large times.

First, we estimate the value $t\|u(t)-v(t)\|_{V}$ uniformly on the interval $[1, \infty)$. Second, the convergence rate is of the order $O(\varepsilon)$. Third, the estimate holds uniformly for $u^{0}$ in bounded subsets of $D(A)$.

We do not obtain the convergence rate $O(\varepsilon)$ (uniformly for bounded subsets in $D(A)$ ) for small times. On the other hand, it is not clear if the weaker estimates from [9] or [6] are optimal. This question is, however, not the purpose of this article.

It is not possible to estimate $t\|u(t)-v(t)\|_{V}$ uniformly on $[1, \infty)$ for an arbitrary choice of initial values $u^{0}=u(0)=v(0) \in V$ and $u^{1}=u^{\prime}(0) \in H$. Theorem 1.1 fails for example for $A=0, u^{0} \in V$, and $u^{1} \in H$ different from 0 . In this case,

$$
\left\|u_{\varepsilon}(t)-v(t)\right\|_{H}=\varepsilon\left(1-e^{-t / \varepsilon}\right)\left\|u^{1}\right\|_{H} \rightarrow \varepsilon\left\|u^{1}\right\|_{H} \quad \text { as } t \rightarrow \infty
$$

3.2. If the initial value $u^{0}$ is more regular in the sense that $u^{0} \in D\left(A^{\alpha}\right)$ for some $\alpha>1$, then, under the assumptions of Theorem 1.1,

$$
\forall t \geq 1 \quad\left\|u_{\varepsilon}(t)-v(t)\right\|_{D\left(A^{\alpha-1 / 2}\right)} \leq \frac{C \varepsilon}{t}\left(\left\|u^{0}\right\|_{D\left(A^{\alpha-1 / 2}\right)}+\sqrt{\varepsilon}\left\|u^{0}\right\|_{D\left(A^{\alpha}\right)}\right)
$$

where $C \geq 0$ is a constant independent of $\varepsilon \in(0,1]$ and $u^{0} \in D\left(A^{\alpha}\right)$. 
3.3. It follows from the proof of Theorem 1.3 that for every $k \geq 0$

$$
\limsup _{t \rightarrow \infty} \sup _{\varepsilon \in(0,1]} \sup _{\substack{u^{0} \in D\left(A^{k}\right) \\\left\|u^{0}\right\|_{D\left(A^{k}\right)} \leq 1}} \frac{t}{\varepsilon}\left\|u_{\varepsilon}(t)-v(t)\right\|_{H}>0 .
$$

In order to see this, one may again assume that $A$ is a multiplication operator and observe that the norms $\|\cdot\|_{D\left(A^{k}\right)}$ are equivalent to the norm $\|\cdot\|_{H}$ on the subspace

$$
\left\{u^{0} \in L^{2}(E ; d \mu): \operatorname{supp} u^{0} \subset\{a(x) \leq 1\}\right\} .
$$

3.4. We may apply our abstract results to the dissipative wave equation

$$
\begin{cases}\varepsilon u_{t t}+u_{t}-\Delta u=0 & \text { in } \boldsymbol{R}_{+} \times \Omega, \\ u=0 & \text { in } \boldsymbol{R}_{+} \times \partial \Omega, \\ u(0, \cdot)=u^{0}, u_{t}(0, \cdot)=\Delta u^{0}, & \end{cases}
$$

and to the heat equation

$$
\begin{cases}v_{t}-\Delta v=0 & \text { in } \boldsymbol{R}_{+} \times \Omega, \\ v=0 & \text { in } \boldsymbol{R}_{+} \times \partial \Omega, \\ v(0, \cdot)=u^{0} & \end{cases}
$$

in a domain $\Omega \subset \boldsymbol{R}^{n}$. In fact, these equations are special cases of equations (1.1) and (1.2) if $A:=-\Delta_{\Omega}^{D}$ is the Dirichlet-Laplace operator on $H=L^{2}(\Omega)$ with domain

$$
D(A):=\left\{u \in H_{0}^{1}(\Omega):-\Delta u \in L^{2}(\Omega)\right\} .
$$

Note that $D\left(A^{1 / 2}\right)=H_{0}^{1}(\Omega)$, and if $\Omega$ has a smooth boundary, then $D(A)=H^{2}(\Omega) \cap H_{0}^{1}(\Omega)$. We thus obtain the following corollary of Theorems 1.1 and 1.3 .

Corollary 3.1. Let $u_{\varepsilon}$ and $v$ be the solutions of the equations (3.1) and (3.2), respectively. There exists a constant $C \geq 0$ independent of $u^{0} \in D(A)$ and $\varepsilon \in(0,1]$ such that for every $t \geq 1$

$$
\frac{t}{\varepsilon}\left\|u_{\varepsilon}(t)-v(t)\right\|_{H_{0}^{1}(\Omega)} \leq C\left(\left\|u^{0}\right\|_{H_{0}^{1}(\Omega)}+\sqrt{\varepsilon}\left\|u^{0}\right\|_{D(A)}\right) .
$$

This estimate is optimal if $\Omega$ contains balls of arbitrarily large radius, e.g. if $\Omega$ is an exterior domain.

Remark 3.2. The estimate in the above Corollary 3.1 is not optimal if $\Omega$ is a bounded domain, or, more generally, if $\Omega$ is a domain for which the first Poincaré inequality 


$$
\int_{\Omega} u^{2} \leq c \int_{\Omega}|\nabla u|^{2}
$$

holds for all $u \in H_{0}^{1}(\Omega)$ and for a uniform constant $c \geq 0$.

In fact, the first Poincare inequality holds if and only if the DirichletLaplace operator is a strictly positive operator on $L^{2}(\Omega)$. Thus, if the first Poincaré inequality holds, then, by Theorem 1.2,

$$
\forall t \geq 1 \quad \frac{e^{\delta t}}{\varepsilon}\left\|u_{\varepsilon}(t)-v(t)\right\|_{H_{0}^{1}(\Omega)} \leq C\left(\left\|u^{0}\right\|_{H_{0}^{1}(\Omega)}+\sqrt{\varepsilon}\left\|u^{0}\right\|_{D(A)}\right) .
$$

3.5. We remark that there are results in the spirit of Theorem 1.1 in the context of nonautonomous, inhomogeneous or nonlinear wave and heat equations; the estimates are, however, only valid on compact time intervals. For results in this direction we refer to H. Bellout and A. Friedman [1], K. J. Engel [3], H. S. Nur [12], H. O. Fattorini [6], B. Najman [10, 11] and B. F. Esham and R. J. Weinacht $[4,5]$. Higher order equations have been studied by J. A. Smoller [14].

\section{References}

[ 1 ] Bellout, H. and Friedman, A., Blow-up estimates for a nonlinear hyperbolic heat equation, SIAM J. Math. Anal., 20 (1989), 354-366.

[2] Chill, R. and Haraux, A., An optimal estimate for the difference of solutions of two abstract evolution equations, J. Differential Equations, 193 (2003), 385-395.

[ 3 ] Engel, K. J., On singular perturbations of second order Cauchy problems, Pacific J. Math., 152 (1992), 79-91.

[4] Esham, B. F. and Weinacht, R. J., Hyperbolic-parabolic singular perturbations for scalar nonlinearities, Appl. Anal., 29 (1988), 19-44.

[ 5 ] Esham, B. F. and Weinacht, R. J., Hyperbolic-parabolic singular perturbations for quasilinear equations, SIAM J. Math. Anal., 20 (1989), 1344-1365.

[6] Fattorini, H. O., Second Order Linear Differential Equations in Banach Spaces, NorthHolland, Amsterdam, 1985.

[7] Ikehata, R., $L^{2}$-convergence results for linear dissipative wave equations in unbounded domains, Asymptotic Anal., 36 (2003), 63-74.

[8] Ikehata, R. and Matsuyama, T., $L^{2}$-behaviour of solutions to the linear heat and wave equations in exterior domains, Sci. Math. Japon., 55 (2002), 33-42.

[9] Kisynski, J., Sur les équations hyperboliques avec petit paramètre, Colloq. Math., 10 (1963), 331-343.

[10] Najman, B., Time singular limit of semilinear wave equations with damping, J. Math. Anal. Appl., 174 (1993), 95-117.

[11] Najman, B., Hyperbolic singular perturbation of a quasilinear parabolic equation, Evolution equations, control theory, and biomathematics (Han sur Lesse, 1991), Dekker, New York, 1994, pp. 405-416.

[12] Nur, H. S., Singular perturbations of differential equations in abstract spaces, Pacific J. Math., 36 (1971), 775-780. 
[13] Reed, M. and Simon, B., Methods of Modern Mathematical Physics, I: Functional Analysis. Second edition, Academic Press, New York, 1980.

[14] Smoller, J. A., Singular perturbations and a theorem of Kisynski, J. Math. Anal. Appl., 12 (1965), 105-114.

\author{
nuna adreso: \\ Ralph Chill \\ Abteilung Angewandte Analysis \\ Universität Ulm \\ D-89069 Ulm \\ Germany \\ E-mail: chill@mathematik.uni-ulm.de
}

Alain Haraux

Université Paris VI

Laboratoire Jacques-Louis Lions

Tour 55-65 5ème étage, 4 pl. Jussieu

75252 Paris Cedex 05

France

E-mail: haraux@ann.jussieu.fr

(Ricevita la 12-an de marto, 2003)

(Reviziita la 29-an de julio, 2003) 